\title{
SPC18 Expression Is an Independent Prognostic Indicator of Patients with Esophageal Squamous Cell Carcinoma
}

\author{
Yuji Yamamoto ${ }^{a, b}$ Naohide Oue ${ }^{a}$ Ryuichi Asai ${ }^{a, c}$ Narutaka Katsuya ${ }^{a}$ \\ Naohiro Uraoka ${ }^{a}$ Naoya Sakamoto ${ }^{a}$ Kazuhiro Sentani ${ }^{a}$ Kazuaki Tanabe $^{b}$ \\ Hideki Ohdan ${ }^{\text {b }}$ Wataru Yasui ${ }^{a}$ \\ a Department of Molecular Pathology, Graduate School of Biomedical and Health Sciences, Hiroshima University, \\ Hiroshima, Japan; ${ }^{b}$ Department of Gastroenterological and Transplant Surgery, Graduate School of Biomedical and \\ Health Sciences, Hiroshima University, Hiroshima, Japan; ' Department of Surgical Oncology, Graduate School of \\ Medicine, Gifu University, Gifu, Japan
}

\section{Keywords}

$\mathrm{SPC} 18 \cdot \mathrm{SEC} 11 \mathrm{~A} \cdot \mathrm{EGFR} \cdot$ Esophageal cancer $\cdot \mathrm{TGF}-\mathrm{a} \cdot \mathrm{Akt}$

\begin{abstract}
Objectives: Esophageal cancer is the sixth most common malignancy worldwide. Signal peptidase complex 18(SPC18) protein, which is encoded by the SEC11A gene, is one of the subunits of the signal peptidase complex and plays an important role in the secretion of proteins including transforming growth factor a (TGF-a). In this study, we investigated the significance of SPC18 expression in human esophageal squamous cell carcinoma (ESCC). Methods: SPC18 expression was examined by immunohistochemistry. RNA interference was used to inhibit SPC18 expression in ESCC cell lines. To examine cell viability, we performed 3-(4,5-dimethylthiazol-2-yl)-2,5-diphenyltetrazolium bromide assays. The effects of SPC18 inhibition on epidermal growth factor receptor (EGFR) signaling were analyzed by Western blot. Results: In total, 46 (50\%) of 92 ESCC cases were positive for SPC18. SPC18 staining was observed more frequently in stage II/III/ IV cases than in stage I cases ( $p=0.028)$. We found that SPC18 expression was significantly associated with increased cancer-specific mortality ( $p=0.006$, log-rank test). SPC18 ex-
\end{abstract}

pression was frequently found in EGFR-positive cases compared with EGFR-negative cases. Cell proliferation and EGFR signaling were inhibited by SPC18 knockdown. Conclusion: Specific inhibitors of SPC18 may be promising anticancer drugs for patients with ESCC.

(c) 2020 S. Karger AG, Basel

\section{Introduction}

Esophageal cancer is the sixth most common malignancy worldwide [1]. The two predominant forms of esophageal cancer are esophageal squamous cell carcinoma (ESCC) and adenocarcinoma. Globally, ESCC accounts for $>90 \%$ of esophageal cancer. Most ESCC is diagnosed at an advanced stage, and even superficial ESCC that appears to extend no further than the submucosa metastasizes to the lymph nodes in $50 \%$ of cases [2]. For localized ESCC, surgery is the primary therapeutic option. However, the prognosis is unsatisfactory, even in curatively resected patients, and the 5-year survival rate is $<50 \%$ after surgery [3]. Several prognostic markers, such as nodal status and tumor stage, are currently accepted for clinical use, and we also previously identified several karger@karger.com

www.karger.com/pat

Karger $\stackrel{2}{\circ}$
(C) 2020 S. Karger AG, Basel

(n)
Naohide Oue

Department of Molecular Pathology, Graduate School of Biomedical and Health Sciences, Hiroshima University, 1-2-3 Kasumi, Minami-ku Hiroshima 734-8551 (Japan)

naoue@ hiroshima-u.ac.jp 
ESCC-associated genes [4-6]. However, these genes cannot completely identify which patients are at a low or high risk for disease recurrence. Therefore, there is an urgent need for new prognostic markers and therapeutic targets for ESCC.

Transforming growth factor (TGF)- $\alpha$ is a mitogenic polypeptide that has a wide range of biological activities [7]. TGF- $\alpha$ activates epidermal growth factor receptor (EGFR) and stimulates multiple signaling pathways involved in cell proliferation, anti-apoptosis, and other processes [8]. Previously, we reported that signal peptidase complex 18 (SPC18) induces TGF-a secretion in human gastric cancer cells [9]. SPC18 protein, which is encoded by the SEC11A gene, is one of the subunits of the signal peptidase complex. Most secretory proteins contain amino terminal or internal signal peptides that direct their sorting to the endoplasmic reticulum (ER) [10]. From the ER, proteins are transported to either the extracellular space or plasma membrane. The ER signal peptides are then cleaved by the signal peptidase complex. It has been reported that the signal peptidase complex purified from canine microsomes has 5 distinct subunits [11]. Two of these subunits, SPC18 and SPC21, are presumed to have catalytic activity [12], indicating that SPC18 overexpression induces TGF- $\alpha$ secretion. SPC18 overexpression is also associated with tumor progression in colorectal and urinary bladder cancer $[13,14]$. These data support the notion that SPC18 protein may be a potential novel marker for a wide variety of malignancies. However, expression of SPC18 has not been examined in ESCC.

In this study, we analyzed the expression and distribution of SPC18 protein in human ESCC by immunohistochemistry and examined the relationship between SPC18 protein expression and clinicopathological characteristics. We also examined the association between EGFR and SPC18 expression, and the effect of inhibiting SPC18 expression by RNA interference (RNAi) on cell growth, invasiveness, and EGFR signaling in ESCC cells.

\section{Materials and Methods}

\section{Tissue Samples}

In a retrospective study design, 92 primary tumors were collected from patients diagnosed with ESCC who underwent surgery between April 2008 and March 2013 at Hiroshima University Hospital (Hiroshima, Japan). All patients underwent curative resection. Only patients without preoperative radiotherapy or chemotherapy and without clinical evidence of distant metastasis were enrolled in the study. Operative mortality was defined as death within 30 days of the patient leaving the hospital and these cases were excluded from the analysis. Postoperative follow-up was scheduled for every 1, 2, or 3 months during the first 2 years after surgery, and every 6 months thereafter, unless more frequent follow-ups were deemed necessary. Chest X-ray, chest computed tomography scanning, and serum chemistry analyses were performed at each follow-up visit. Follow-up was conducted by the physician until the patient's death or the date of the last documented contact.

Archival formalin-fixed, paraffin-embedded tissues from 92 patients who had undergone surgical excision for ESCC were examined by immunohistochemical analysis. One or two representative tumor blocks, including the tumor center, invading front, and tumor-associated nonneoplastic mucosa, were examined from each patient by immunohistochemistry. In cases of large, late-stage tumors, two different sections were examined to include representative areas of the tumor center as well as the lateral and deep tumor invasive front. Tumor staging was determined according to the TNM classification system [15].

\section{Immunohistochemistry}

Immunohistochemical analysis was performed with a Dako Envision+ rabbit or mouse peroxidase detection system (Dako Cytomation, Carpinteria, CA, USA). Antigen retrieval was conducted by microwave heating in citrate buffer $(\mathrm{pH}$ 6.0) for $30 \mathrm{~min}$. Peroxidase activity in sections was blocked by incubation with $3 \%$ $\mathrm{H}_{2} \mathrm{O}_{2}$ in methanol for $10 \mathrm{~min}$, followed by incubation with normal goat serum (Dako Cytomation) for 20 min to block nonspecific antibody-binding sites. Sections were incubated with an antiSPC18 antibody [9] or anti-EGFR antibody (1:20; Novocastra, Newcastle, UK) for $1 \mathrm{~h}$ at room temperature, followed by incubation with Envision+ anti-rabbit or anti-mouse peroxidases for $1 \mathrm{~h}$. For color development, sections were incubated with DAB substrate-chromogen solution (Dako Cytomation) for $10 \mathrm{~min}$. Sections were counterstained with $0.1 \%$ hematoxylin. Negative controls were prepared by omission of the primary antibody.

The expression of SPC18 and EGFR was scored in all tumors as positive or negative. When $>10 \%$ of tumor cells were stained, the immunostaining was considered as positive. Using this definition, 2 surgical pathologists (N.O. and K.S.), without knowledge of the clinical and pathological parameters or patients' outcomes, independently reviewed the immunoreactivity of each specimen. Interobserver differences were resolved by consensus under a double-headed microscope after the independent review.

\section{Cell Lines}

Four cell lines derived from human esophageal cancer (TE-1, TE-5, TE-10, and TE-11) were purchased from the Japanese Collection of Research Bioresources Cell Bank (Osaka, Japan). Cell line identity was verified by short tandem repeat profiling (Promega, Madison, MD, USA). All cell lines were maintained in RPMI1640 (Nissui Pharmaceutical Co., Ltd., Tokyo, Japan) containing 10\% FBS (BioWhittaker, Walkersville, MD, USA) in a humidified atmosphere with $5 \% \mathrm{CO}_{2}$ at $37^{\circ} \mathrm{C}$.

\section{Western Blot Analysis}

Cells were lysed as described previously [16]. The lysates (40 $\mu \mathrm{g}$ protein) were solubilized in Laemmli sample buffer by boiling, and then subjected to $10 \%$ SDS-PAGE followed by electrotransfer onto a nitrocellulose membrane. Western blot procedures were performed as described previously [16]. An anti-SPC18 antibody was prepared as described previously [9]. Anti-Akt, anti-phosphory- 
Fig. 1. Expression of SPC18 in ESCC. a Immunostaining of SPC18 in ESCC. $\times 10$. b Immunostaining of SPC18 in ESCC. $\times 400$. c Immunostaining of EGFR in the same ESCC case shown in b. $\times 400$. d Kaplan-Meier plot of ESCC patient survival according to SPC18 expression.

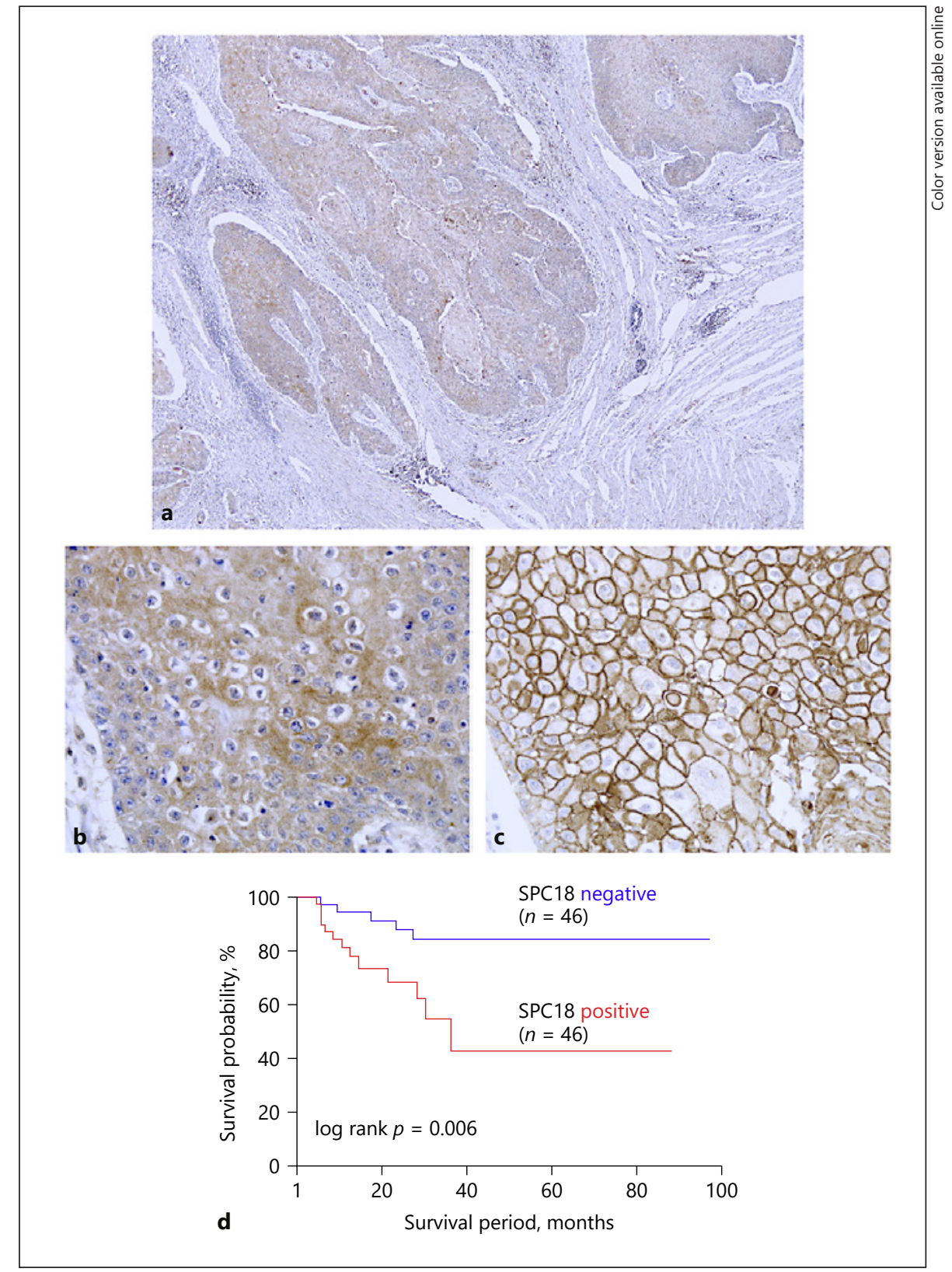

lated Akt (Ser473), anti-Erk, and anti-phosphorylated Erk1/2 (Thr202/Tyr204) antibodies were purchased from Cell Signaling Technology (Danvers, MA, USA). $\beta$-Actin (Sigma Chemical, St. Louis, MO, USA) was also stained as a loading control.

RNAi and Cell Proliferation Assay

Short interfering RNA (siRNA) oligonucleotides targeting SPC18 and a negative control were purchased from Invitrogen (Carlsbad, CA, USA). We used 3 independent SPC18 siRNA oligonucleotide sequences. Transfection was performed using Lipofectamine RNAiMAX (Invitrogen) as described previously [17]. Briefly, 60 pmol siRNA and $10 \mu \mathrm{L}$ Lipofectamine RNAiMAX were mixed in $1 \mathrm{~mL}$ RPMI1640 medium (10 nmol/L final concentra- tion). After 20 min of incubation, the mixture was added to the cells, and then the cells were plated in culture dishes. At $48 \mathrm{~h}$ after transfection, the cells were analyzed.

Cell proliferation was monitored after 1, 2, and 4 days by 3-(4,5-dimethylthiazol-2-yl)-2,5-diphenyltetrazolium bromide (MTT) assay as described previously [9]. Three independent experiments were carried out. The mean \pm standard error (SE) was calculated for each experiment.

\section{Measurement of TGF- $\alpha$}

The levels of TGF- $\alpha$ in culture media were measured by enzyme-linked immunosorbent assay (ELISA). The TGF- $\alpha$ ELISA kit was purchased from R\&D Systems. Forty-eight hours after siRNA 
transfection, cells were serum-starved for $72 \mathrm{~h}$ in serum-free RPMI1640, during which time the media were changed every 24 h. Cell culture media were collected, and cells were centrifuged at $1,300 \mathrm{rpm}$ for $10 \mathrm{~min}$. Pellets were discarded, and the supernatant was tested for levels of TGF- $\alpha$ as per the manufacturer's instructions.

\section{Statistical Methods}

Associations between clinicopathological parameters and SPC18 expression were analyzed by Fisher's exact test. KaplanMeier survival curves were constructed for SPC18-positive and SPC18-negative patients. Differences between survival curves were tested for statistical significance by the log-rank test. Univariate and multivariate Cox regressions were used to evaluate the associations between clinical covariates and cancer-specific mortality. The hazard ratio (HR) and 95\% confidence interval (CI) were estimated from Cox proportional hazard models. Differences between the 2 groups (SPC18 siRNA-transfected cells and negative-control siRNA-transfected cells) were tested by Student's $t$ test. A $p$ value $<0.05$ was considered to be statistically significant.

\section{Results}

\section{Expression and Distribution of SPC18 Protein in} ESCC Tissues

Immunohistochemical analysis was performed using whole paraffin-embedded blocks to analyze in detail the expression and distribution of SPC18 protein in ESCC tissues. In nonneoplastic esophageal mucosa, staining of SPC18 was either weak or absent in the epithelial and stromal cells, but the corresponding ESCC tissue showed relatively stronger, more extensive staining (Fig. 1a). SPC18 was detected in the cytoplasm of tumor cells (Fig. 1b). The percentage of SPC18-stained tumor cells ranged from 0 to $90 \%$. When $>10 \%$ of tumor cells were stained, the immunostaining was considered as positive for SPC18. In total, 46 (50\%) of 92 ESCC cases were positive for SPC18. SPC18 staining was observed more frequently in stage II/III/IV cases than in the stage I cases $(p=0.028$; Fisher's exact test; Table 1$)$. We found that SPC18 expression was significantly associated with increased cancer-specific mortality $(p=0.006$; log-rank test; Fig. 1d).

Next, we analyzed the expression of EGFR by immunohistochemistry. The percentage of EGFR-stained tumor cells ranged from 0 to $90 \%$. When $>10 \%$ of tumor cells were stained, the immunostaining was considered positive for EGFR. In total, 19 (21\%) of 92 ESCC cases were positive for EGFR. SPC18 expression was found more frequently in EGFR-positive cases than in EGFRnegative cases (Table 1). In both SPC18- and EGFR-pos-
Table 1. Relationship between SPC18 expression and clinicopathological characteristics of ESCC patients

\begin{tabular}{|c|c|c|c|}
\hline & \multicolumn{2}{|c|}{ SPC18 expression } & \multirow[t]{2}{*}{$p$ value $^{\mathrm{a}}$} \\
\hline & positive & negative & \\
\hline \multicolumn{4}{|l|}{ Age } \\
\hline$<65$ years & $19(21 \%)$ & 19 & \multirow[t]{2}{*}{1.000} \\
\hline$\geq 65$ years & $27(29 \%)$ & 27 & \\
\hline \multicolumn{4}{|l|}{ Sex } \\
\hline Male & $42(46 \%)$ & 37 & \multirow[t]{2}{*}{0.135} \\
\hline Female & $4(4 \%)$ & 9 & \\
\hline \multicolumn{4}{|l|}{ T classification } \\
\hline T1a & $3(3 \%)$ & 11 & \multirow[t]{2}{*}{0.020} \\
\hline $\mathrm{T} 1 \mathrm{~b} / 2 / 3 / 4$ & $43(47 \%)$ & 35 & \\
\hline \multicolumn{4}{|l|}{$\mathrm{N}$ classification } \\
\hline N0 & $16(17 \%)$ & 25 & \multirow[t]{2}{*}{0.059} \\
\hline $\mathrm{N} 1 / 2 / 3$ & $30(33 \%)$ & 21 & \\
\hline \multicolumn{4}{|l|}{ Stage } \\
\hline I & $11(12 \%)$ & 21 & \multirow[t]{2}{*}{0.028} \\
\hline II/III/VI & $35(38 \%)$ & 25 & \\
\hline \multicolumn{4}{|l|}{ Lymphatic invasion } \\
\hline Negative & $15(16 \%)$ & 17 & \multirow[t]{2}{*}{0.662} \\
\hline Positive & $31(34 \%)$ & 29 & \\
\hline \multicolumn{4}{|l|}{ Vascular invasion } \\
\hline Negative & $29(32 \%)$ & 34 & \multirow[t]{2}{*}{0.262} \\
\hline Positive & $17(18 \%)$ & 12 & \\
\hline \multicolumn{4}{|c|}{ Histological classification } \\
\hline Well, moderately & $36(39 \%)$ & 32 & \multirow[t]{2}{*}{0.342} \\
\hline poorly & $10(11 \%)$ & 14 & \\
\hline \multicolumn{4}{|l|}{ EGFR expression } \\
\hline Negative & $33(36 \%)$ & 40 & \multirow[t]{2}{*}{0.068} \\
\hline Positive & $13(14 \%)$ & 6 & \\
\hline
\end{tabular}

itive cases, SPC18-stained ESCC cells were also stained for EGFR (Fig. 1b, c). However, no statistically significant association was found between SPC18 and EGFR expression ( $p=0.068$; Fisher's exact test; Table 1$)$.

The univariate analysis indicated that expression of SPC18 (HR 3.85; 95\% CI 1.43-12.12; $p=0.006$ ), T classification (HR 7.44; 95\% CI 2.41-32.57; $p=0.001$ ), N classification (HR 19.88; 95\% CI 4.05-359; $p=0.001$ ), lymphatic invasion (HR 5.04; 95\% CI 1.43-32.02; $p=0.009$ ), vascular invasion (HR 4.37; 95\% CI 1.69-12.24; $p=0.002$ ), and EGFR expression (HR 3.01; 95\% CI 1.11-7.67; $p=$ 0.032 ) were associated with survival, but age, sex, and histological classification were not (Table 2). Moreover, in the multivariate model, SPC18 expression (HR 3.17; 95\% CI 1.14-10.46; $p=0.027$ ) and $\mathrm{N}$ classification (HR 9.35; $95 \%$ CI 1.45-190; $p=0.015)$ were independent prognostic indicators (Table 2). 
Table 2. Univariate and multivariate Cox regression analyses of SPC18 expression and survival of ESCC patients

\begin{tabular}{|c|c|c|c|c|}
\hline \multirow[t]{2}{*}{ Characteristic } & \multicolumn{2}{|l|}{ Univariate analysis } & \multicolumn{2}{|l|}{ Multivariate analysis } \\
\hline & HR (95\% CI) & $p$ value & HR (95\% CI) & $p$ value \\
\hline \multicolumn{5}{|l|}{ Age } \\
\hline$\leq 65$ years & 1 (ref.) & 0.365 & & \\
\hline$>65$ years & $0.65(0.25-1.67)$ & & & \\
\hline \multicolumn{5}{|l|}{ Sex } \\
\hline Female & 1 (ref.) & 0.138 & & \\
\hline Male & $3.51(0.72-63.3)$ & & & \\
\hline \multicolumn{5}{|l|}{ T classification } \\
\hline $\mathrm{T} 1$ & 1 (ref.) & 0.002 & 1 (ref.) & 0.331 \\
\hline $\mathrm{T} 2 / 3 / 4$ & $7.44(2.41-32.54)$ & & $2.07(0.50-11.42)$ & \\
\hline \multicolumn{5}{|l|}{$\mathrm{N}$ classification } \\
\hline N0 & 1 (ref.) & 0.001 & 1 (ref.) & 0.015 \\
\hline $\mathrm{N} 1 / 2 / 3$ & $19.88(4.05-99.21)$ & & $9.35(1.45-190.84)$ & \\
\hline \multicolumn{5}{|l|}{ Lymphatic invasion } \\
\hline Negative & 1 (ref.) & 0.009 & 1 (ref.) & 0.706 \\
\hline Positive & $5.04(1.43-32.02)$ & & $1.41(0.25-11.23)$ & \\
\hline \multicolumn{5}{|l|}{ Vascular invasion } \\
\hline Negative & 1 (ref.) & & 1 (ref.) & 0.592 \\
\hline Positive & $4.37(1.69-12.27)$ & 0.002 & $1.39(0.42-5.34)$ & \\
\hline \multicolumn{5}{|c|}{ Histological classification } \\
\hline Well, moderately & 1 (ref.) & & & \\
\hline poorly & $0.83(0.28-2.23)$ & 0.733 & & \\
\hline \multicolumn{5}{|l|}{ EGFR expression } \\
\hline Negative & 1 (ref.) & & 1 (ref.) & \\
\hline Positive & $3.01(1.11-7.67)$ & 0.031 & $2.65(0.91-7.36)$ & 0.072 \\
\hline \multicolumn{5}{|l|}{ SPC18 expression } \\
\hline Negative & 1 (ref.) & & 1 (ref.) & \\
\hline Positive & $3.85(1.43-12.13)$ & 0.006 & $3.17(1.14-10.46)$ & 0.027 \\
\hline
\end{tabular}

HR, hazard ratio; CI, confidence interval.

Effect of SPC18 Inhibition on the Proliferation of ESCC Cells

We examined the effect of SPC18 inhibition on cell proliferation by MTT assay. SPC18 protein expression was clearly detected in in the TE-1, TE-5, TE-10, and TE-11 cell lines (Fig. $2 \mathrm{a}$ ). Western blot showed that the expression of SPC18 in both TE- 1 and TE- 5 cells was substantially suppressed by treatment with siRNA1 and siRNA3 (Fig. 2b, c). Thus, siRNA1 and siRNA3 were used for the following experiments. Cell proliferation was analyzed by MTT assay. SPC18 siRNA1- and siRNA3-transfected TE-1 cells showed significantly reduced cell proliferation relative to negative-control siRNA-transfected TE- 1 cells (Fig. 2d). We also performed an MTT assay at 4 days after siRNA transfection of TE- 5 cells, and similar results were obtained (Fig. 2e).
Effect of SPC18 inhibition on EGFR signaling was examined because SPC18 induces TGF- $\alpha$ secretion [9]. It is well known that TGF- $\alpha$ phosphorylates EGFR, which subsequently stimulates multiple signaling pathways involved in cellular proliferation, antiapoptosis, and other cellular processes. We analyzed the phosphorylation of Akt and Erk in TE- 5 cells with SPC18 inhibition. The levels of phosphorylated Akt and Erk in cells transfected with SPC18 siRNA1 and siRNA3 were lower than those in cells transfected with negative-control siRNA (Fig. 3).

Previously, we reported that the levels of TGF- $\alpha$ in culture media from gastric cancer cells are reduced by knockdown of SPC18. Thus, effect of SPC18 inhibition on TGF- $\alpha$ secretion was examined by ELISA in TE- 5 cells. However, TGF- $\alpha$ was not detected in culture media from the TE-5 cells (data not shown). 
Fig. 2. Effect of SPC18 inhibition on ESCC cell lines. a Western blot analysis of SPC18 in esophageal cancer cell lines. b Western blot analysis of SPC18 in TE-1 cells transfected with negative-control siRNA or SPC18 siRNAs. c Western blot analysis of SPC18 in TE-5 cells transfected with negative-control siRNA or SPC18 siRNAs. d Effect of SPC18 knockdown on TE-1 cell proliferation. Cell proliferation was assessed by MTT assay at days 1, 2, and 4 after seeding in 96-well plates. e Effect of SPC18 knockdown on TE- 5 cell proliferation. Cell proliferation was assessed by MTT assay at days 1, 2, and 4 after seeding in 96-well plates.

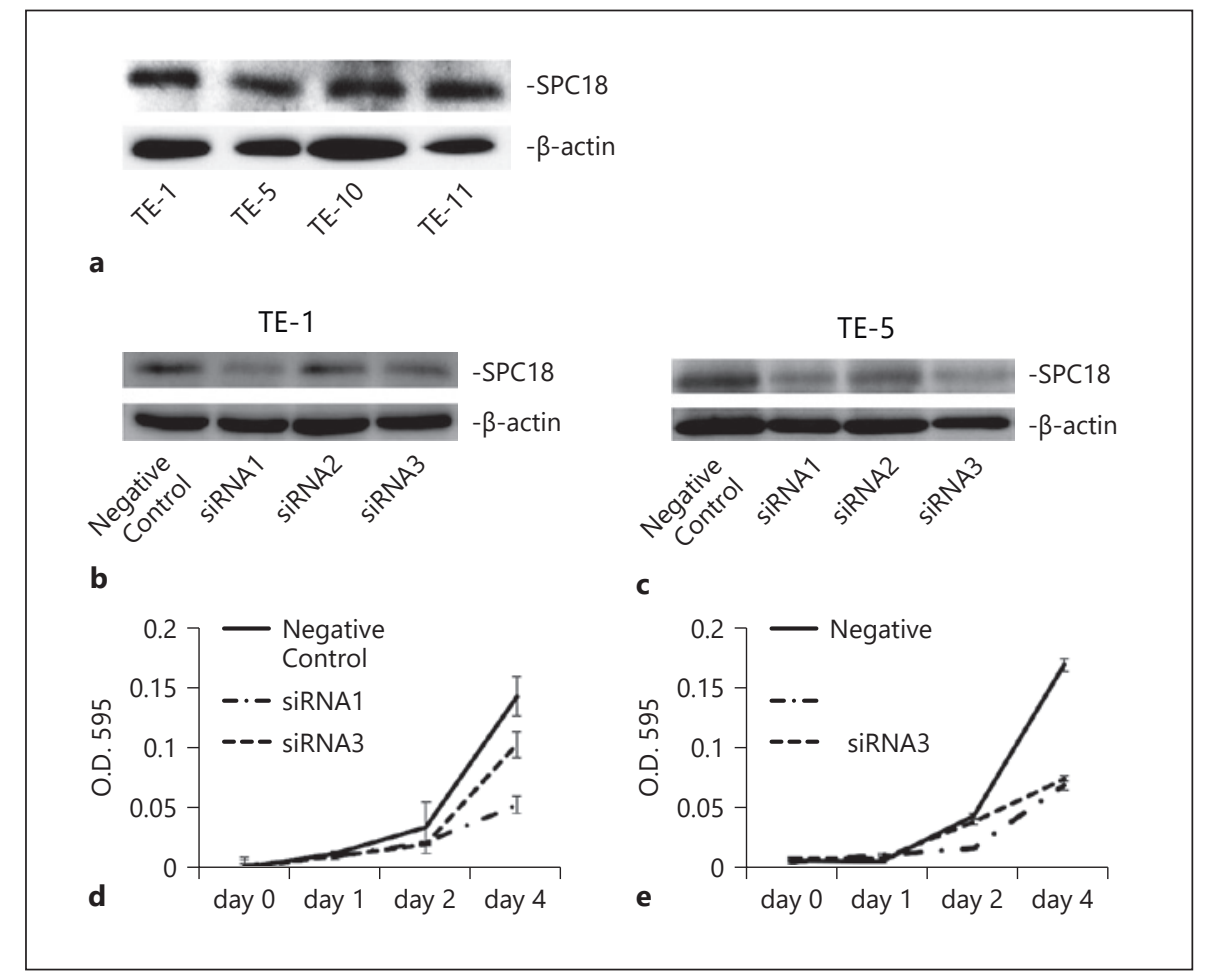

\section{Discussion}

In this study, we analyzed the expression and distribution of SPC18 protein in human ESCC by immunostaining. In nonneoplastic esophagus, weak or no staining of SPC18 was observed in epithelial and stromal cells whereas ESCC cells showed stronger, more extensive staining. The percentage of SPC18-stained tumor cells ranged from 0 to $90 \%$, and $50 \%$ of the ESCC cases were positive for SPC18. SPC18 staining was observed more frequently in stage II/III/IV cases than in stage I cases. These results indicate that SPC18 plays a major role in the progression of ESCC. Furthermore, multivariate analysis demonstrated that SPC18 expression is an independent prognostic indicator. In addition to clinical stage information, immunohistochemical analysis of SPC18 can facilitate prediction of patient survival.

We have reported overexpression of SPC18 in gastric, colorectal, and urinary bladder cancer $[9,13,14]$. SPC18 expression is correlated with patient survival in these cancers. SPC18 is also upregulated in ovarian cancer, and SPC18 expression is associated with tumor progression and patient survival. These results suggest that SPC18 participates in the malignant behaviors of a wide variety of human cancers.

SPC18 Expression in Esophageal Cancer

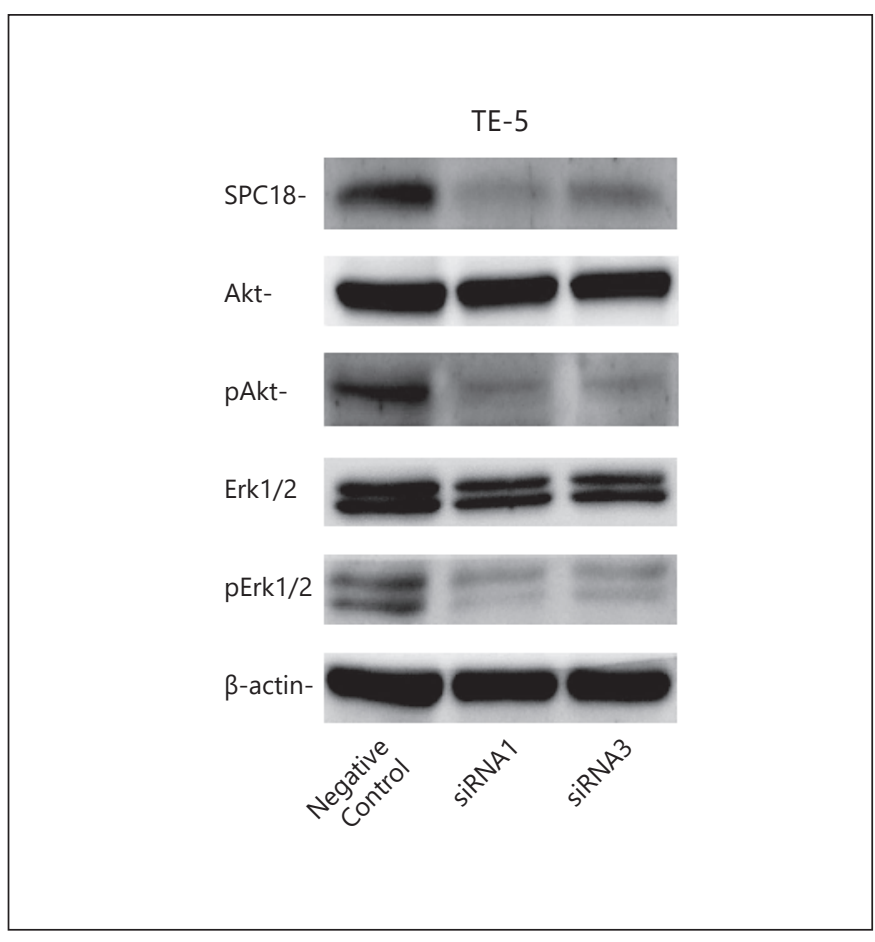

Fig. 3. Western blot analysis of SPC18, Akt, phosphorylated Akt (pAkt), Erk1/2, and phosphorylated Erk1/2 (pErk1/2) in lysates of TE- 5 cells transfected with negative-control siRNA or SPC18 siRNAs. 
Functional analysis of SPC18 is important to understand tumor progression. In this study, we showed that inhibition of SPC18 reduced cell proliferation. SPC18 protein is one of the subunits of the signal peptidase complex [10]. Increased activity of the signal peptidase complex caused by SPC18 protein overexpression induces the secretion of several kinds of growth factors including TGF- $\alpha$ [9]. However, TGF- $\alpha$ was not detected in culture media from the TE- 5 cells. We confirmed that the levels of phosphorylated Akt and Erk, both activated by EGFR, were reduced by SPC18 knockdown, suggesting that other growth factors may activate EGFR. These results suggest that SPC18 is involved in the activation of EGFR signaling in ESCC cells.

Because SPC18 knockdown reduced cell proliferation, we did not analyze the effect of SPC18 knockdown on invasive activity. We found that the levels of phosphorylated Akt and Erk were reduced by SPC18 knockdown. It has been reported that phosphorylated Akt and Erk increase the invasive activity of ESCC cells. TRIM44 promotes ESCC progression via the Akt pathway [18]. MicroRNA148a regulates the Erk signaling pathway and suppresses the development of ESCC by targeting MAP3K9 [19]. Taken together, these findings suggest that SPC18 activates ESCC cell invasiveness via the phosphorylation of Akt and Erk.

During the last decade, immunotherapy has induced clinical responses in cancer patients [20]. The discovery of tumor antigens can improve antitumor $\mathrm{T}$ cell responses and T cell-based immunotherapy. In fact, it has been reported that neoantigen-based immunotherapy is effective for lung squamous cell carcinoma [21]. Importantly, one of the signal peptidase functions is epitope processing
[22]. Because the expression of SPC18 is upregulated in ESCC cells, several alterations of epitope processing may occur in SPC18-positive cases. Associations between immune responses, like lymphocytic cell infiltration, and SPC18 expression, should be analyzed in the future.

In summary, we demonstrated that SPC18 expression is associated with the tumor stage and patient survival. We also found that SPC18 knockdown inhibits tumor cell proliferation and phosphorylates Akt and Erk. Specific inhibitors of SPC18 may be promising anticancer drugs for patients with basal-like bladder cancer.

\section{Acknowledgments}

This work was supported by Grants-in-Aid for Scientific Research (B-15H04713) and for Challenging Exploratory Research (26670175 and 16K15247) from the Japan Society for the Promotion of Science and the Takeda Science Foundation. We thank the Edanz Group (www.edanzediting.com) for editing a draft of this manuscript.

\section{Statement of Ethics}

This study was approved by the Ethics Committee for Human Genome Research of the Hiroshima University (Hiroshima, Japan). Because written informed consent was not obtained, for strict privacy protection, any identifying information for all samples was removed before analysis.

\section{Disclosure Statement}

The authors declare no conflicts of interest.

\section{References}

1 Stoner GD, Gupta A. Etiology and chemoprevention of esophageal squamous cell carcinoma. Carcinogenesis. 2001 Nov;22(11):1737-46.

2 Goseki N, Koike M, Yoshida M. Histopathologic characteristics of early stage esophageal carcinoma. A comparative study with gastric carcinoma. Cancer. 1992 Mar;69(5):1088-93.

3 Courrech Staal EF, van Coevorden F, Cats A, Aleman BM, van Velthuysen ML, Boot $\mathrm{H}$, et al. Outcome of low-volume surgery for esophageal cancer in a high-volume referral center. Ann Surg Oncol. 2009 Dec;16(12):3219-26.

4 Mukai S, Oue N, Oshima T, Mukai R, Tatsumoto Y, Sakamoto N, et al. Overexpression of transmembrane protein BST2 is associated with poor survival of patients with esophageal, gastric, or colorectal cancer. Ann Surg Oncol. 2017 Feb;24(2):594-602.

5 Nishimura T, Tamaoki M, Komatsuzaki R, Oue N, Taniguchi H, Komatsu M, et al. SIX1 maintains tumor basal cells via TGF-beta pathway and associates with poor prognosis in esophageal cancer. Cancer Sci. 2016;17: 13135.

6 Imai T, Oue N, Sentani K, Sakamoto N, Uraoka N, Egi H, et al. KIF11 is required for spheroid formation by oesophageal and colorectal cancer cells. Anticancer Res. 2017 Jan;37(1):47-55.

7 Gangarosa LM, Dempsey PJ, Damstrup L, Barnard JA, Coffey RJ. Transforming growth factor-alpha. Baillieres Clin Gastroenterol. 1996 Mar; 10(1):49-63.

8 Thøgersen VB, Sørensen BS, Poulsen SS, Orntoft TF, Wolf H, Nexo E. A subclass of HER1 ligands are prognostic markers for survival in bladder cancer patients. Cancer Res. 2001 Aug;61(16):6227-33.

9 Oue N, Naito Y, Hayashi T, Takigahira M, Kawano-Nagatsuma A, Sentani K, et al. Signal peptidase complex 18, encoded by SEC11A, contributes to progression via TGF- $\alpha$ secretion in gastric cancer. Oncogene. $2014 \mathrm{Jul}$; 33(30):3918-26.

10 Nickel W, Rabouille C. Mechanisms of regulated unconventional protein secretion. Nat Rev Mol Cell Biol. 2009 Feb;10(2):148-55.

11 Greenburg G, Shelness GS, Blobel G. A subunit of mammalian signal peptidase is homologous to yeast SEC11 protein. J Biol Chem. 1989 Sep;264(27):15762-5.

12 Shelness GS, Blobel G. Two subunits of the canine signal peptidase complex are homologous to yeast SEC11 protein. J Biol Chem. 1990 Jun;265(16):9512-9.

13 Hattori T, Sentani K, Naohide O, Sakamoto N, Yasui W. Clinicopathological significance of SPC18 in colorectal cancer: SPC18 participates in tumor progression. Cancer Sci. 2017 Jan;108(1):143-50. 
14 Shigematsu Y, Oue N, Sekino Y, Sakamoto N, Sentani K, Uraoka N, et al. SEC11A Expression is associated with basal-like bladder cancer and predicts patient survival. Pathobiology. 2019;86(4):208-16.

15 Sobin LH, Compton CC. TNM seventh edition: what's new, what's changed: communication from the International Union Against Cancer and the American Joint Committee on Cancer. Cancer. 2010;116:5336-9.

16 Yasui W, Ayhan A, Kitadai Y, Nishimura K, Yokozaki $\mathrm{H}$, Ito $\mathrm{H}$, et al. Increased expression of $\mathrm{p} 34 \mathrm{cdc} 2$ and its kinase activity in human gastric and colonic carcinomas. Int J Cancer. 1993 Jan;53(1):36-41.
17 Sakamoto N, Oue N, Sentani K, Anami K, Uraoka N, Naito Y, et al. Liver-intestine cadherin induction by epidermal growth factor receptor is associated with intestinal differentiation of gastric cancer. Cancer Sci. 2012 Sep; 103(9):1744-50.

18 Xiong D, Jin C, Ye X, Qiu B, Jianjun X, Zhu S, et al. TRIM44 promotes human esophageal cancer progression via the AKT/mTOR pathway. Cancer Sci. 2018 Oct;109(10):3080-92.

19 Zhang BX, Yu T, Yu Z, Yang XG. MicroRNA148a regulates the MAPK/ERK signaling pathway and suppresses the development of esophagus squamous cell carcinoma via targeting MAP3K9. Eur Rev Med Pharmacol Sci. 2019 Aug;23(15):6497-504.
20 Christofi T, Baritaki S, Falzone L, Libra M, Zaravinos A. Current perspectives in cancer immunotherapy. Cancers (Basel). 2019 Sep; 11(10):11.

21 Li F, Chen C, Ju T, Gao J, Yan J, Wang P, et al. Rapid tumor regression in an Asian lung cancer patient following personalized neo-epitope peptide vaccination. OncoImmunology. 2016 Oct;5(12):e1238539.

22 Leclerc M, Mezquita L, Guillebot De Nerville G, Tihy I, Malenica I, Chouaib S, et al. Recent advances in lung cancer immunotherapy: input of T-cell epitopes associated with impaired peptide processing. Front Immunol. 2019 Jul;10:1505. 